\title{
Diabetic cardiomyopathy_What do we know about it?
}

\author{
Muhammad Asrar ul Haq ${ }^{1,2}$, Vivek Mutha ${ }^{1,2}$, Nima Rudd ${ }^{1,2}$, Chiew Wong ${ }^{1,2}$ \\ ${ }^{1}$ Department of Medicine, University of Melbourne, Melbourne, Australia \\ ${ }^{2}$ Department of Cardiology, The Northern Hospital, Melbourne, Australia \\ Email: muhammad.asrar@unimelb.edu.au
}

Received 9 May 2013; revised 12 June 2013; accepted 29 June 2013

Copyright (C 2013 Muhammad Asrar ul Haq et al. This is an open access article distributed under the Creative Commons Attribution License, which permits unrestricted use, distribution, and reproduction in any medium, provided the original work is properly cited.

\begin{abstract}
Diabetic cardiomyopathy is defined as the presence of myocardial dysfunction in patients with diabetes in the absence of coronary artery disease, hypertension, or other known cardiac disease. Diabetes has been shown to affect the heart through various cellular mechanisms leading to enhanced myocardial fibrosis, left ventricular hypertrophy, systolic and diastolic dysfunction. With increasing incidence of type II diabetes mellitus, it has continuously rising health and financial implications in both developed and developing countries. Hyperglycaemia seems to be the main deriving force, and careful glycaemic control as well as early administration of neurohormonal antagonists currently remains the mainstay of therapy. Many newer treatment targets are currently being explored. Here we present a brief review of its pathophysiology, association with heart failure symptoms, and management strategies.
\end{abstract}

Keywords: Cardiomyopathy; Diabetes; Heart Failure

\section{INTRODUCTION}

Diabetic cardiomyopathy is defined as the presence of myocardial dysfunction in patients with diabetes in the absence of coronary artery disease and hypertension, or other known cardiac disease [1]. Diabetes has been shown to affect the heart through various cellular mechanisms leading to enhanced myocardial fibrosis, left ventricular hypertrophy (LVH), systolic and diastolic dysfunction [1]. With increasing incidence of type II diabetes mellitus, it has continuously rising health and financial implications in both developed and developing countries. Hyperglycaemia seems to be the main deriving force [2], and careful glycaemic control as well as early administration of neurohormonal antagonists currently remains the mainstay of therapy.

The risk for heart failure (HF) in diabetics independent of other confounding factors is increased upto 2.4:1 in males and 5:1 in females [3-8]. Elevated glycated haemoglobin (HbA1c) is itself associated with an $8 \%$ increase in HF incidence [9]. More specifically, up to $75 \%$ of asymptomatic normotensive diabetic patients with normal ejection fraction (LVEF) will have diastolic abnormalities [10].

\section{PATHOPHYSIOLOGY}

Angiographic and autopsy studies have confirmed higher prevalence of cardiomyopathy in diabetics when compared to non-diabetic patients, as well as more extensive disease patterns and more severe proximal and distal CAD [11-18]. With similar infarct sizes, diabetic patients have a far greater risk of developing HF post-myocardial infarction (MI) since the compensatory mechanisms to maintain cardiac output in surviving myocardium, eg hyperkinesis to compensate for non viable myocardium, are not as active due to various intra- and extra-myocardial factors and reduced coronary blood flow [19-23]. There is also enhanced thickening of capillary basement membrane, myocellular atrophy and hypertrophy with myocardial and interstitial fibrosis, which further reduce myocardial function [24-26].

\subsection{Hyperglycaemia}

Hyperglycaemia is the main trigger factor. One of the principle abnormalities is impaired coronary vasodilation capacity seconday to increased production of AGEs (advanced glycation end-products). Increased generation of mitochondrial free radicals (Reactive oxygen species; ROS) is another mechanism, which affects the contractility $[27,28]$. Severity of diastolic dysfunction correlates positively with HbA1c levels [9] which may be related to the activity of increased ROS causing increased collagen deposition in the myocardium leading to fibrosis $[29,30]$.

\subsection{Fatty Acids}

In healthy individuals the energy required for cardiac 
function comes from glucose metabolism and free fatty acids (FFAs). Cardiac ischaemia or increased intra-ventricular pressure changes cardiac ATP production to a predominant glucose oxidation [31]. This phenomenon does not occur in diabetic patients and only $10 \%$ of the myocardial energy comes from glucose. This is mainly due to depleted glucose transporter proteins (glucose transporter-1 and -4). This results in a more pronounced beta-oxidation of FFAs [32]. Elevated FFAs are associated with insulin resistance and calcium transporter protein dysfunction, both leading to impaired cardiac function. Increased fatty acids are also associated with the activation of proliferation activated receptor- $\alpha(\operatorname{PPAR} \alpha)$, suspected to promote mitochondrial uncoupling of oxidative phosphorylation [33], a mechanism that reduces myocardial high-energy reserves and contractile dysfunction [34]. Increased intracellular FFAs may directly lead to apoptosis.

\subsection{Protein Kinase $\mathrm{C}$}

Protein kinase $\mathrm{C}$, an intracellular signalling molecule, is activated in diabetes and can lead to endothelial dysfunction by reducing the concentration of nitric oxide and increasing free radical production. Protein kinase $\mathrm{C}$ can also enhance leukocyte adhesion, increase albumin permeability, and impair fibrinolysis [35,36].

\subsection{Renin Angiotensin Aldosterone System (RAAS)}

In a non-diabetic patient RAAS is activated by myocardial stretch owing to the stretch receptors. In diabetic subjects, however, an upregulation of RAAS occurs despite minimal changes in myocardial loading [37]. It has been suggested that aldosterone and glucose can cause cardiac fibrosis through stimulation of myofibroblast growth in patients with a dysregulated RAAS especially with concomitant hyperglycaemia [38].

\subsection{Hypoxia-Inducible Factor-1}

Normally, chronic cardiac ischaemia promotes angiogenesis and collateral vessel formation, mainly through hypoxia-inducible factor-1 (HIF-1), a transcriptional regulator complex present in many gene promoters, including vascular endothelial growth factor (VEGF). In diabetic patients, this angiogenic response to myocardial ischaemia is blunted [39] mainly secondary to markedly reduced (40\% - 70\%) VEGF and its receptors VEGF-R1 and VEGF-R2 as suggested by animal studies.

\subsection{Endothelial Dysfunction}

Hyperglycaemia results in impairment of endothelial cell nitrous oxide (NO) production, increased production of vasoconstrictor prostaglandins, glycated proteins, endothelium adhesion molecules and vascular growth factors, which cumulatively enhance vasomotor tone and vascular permeability, growth and remodelling [35]. Hyperglycaemia also enhances endothelial cell matrix production, which may contribute to basement membrane thickening [40]. All these changes will cause increased atherosclerosis and reduced collateral circulation formation in diabetic patients, which may explain the increased infarct extension and congestive HF after MI in these patients.

\subsection{Arterial Stiffness}

It is well established that hypertension and diabetes lead to increased arterial stiffness [29,41] mainly because of endothelial dysfunction. Reduced compliance of the large arteries in turn affect central systolic pressure and LV afterload, resulting in decreased central diastolic and coronary perfusion pressures [42]. These changes will ultimately result in chronic myocardial ischaemia leading to interstitial fibrosis and HF [43].

\subsection{Autonomic Neuropathy}

Cardiac autonomic neuropathy is associated with diastolic dysfunction in diabetic patients. It starts with an increase in resting heart rate and a loss of heart rate variability. This can influence the chronotropic and inotropic response of the myocardium. Ventricular filling abnormalities are also prevalent in diabetic patients with autonomic neuropathy independent of duration of diabetes, presence of retinopathy, HbA1, or blood glucose levels [44]. A significant correlation has been described between the E/A ratio and autonomic neuropathy [45].

\subsection{Disordered Copper Metabolism}

Alterations in copper metabolism have also been proposed as an important contributor to the development and progression of diabetic cardiomyopathy. Elevated serum copper levels are found in patients with diabetes, and the highest levels are found in those with microvascular complications and hypertension [46]. Hyperglycaemia can damage the copper binding properties of ceruloplasmin and albumin (the main copper binding proteins in plasma), resulting in increased copper levels in the extracellular matrix $[47,48]$. Increased copper in the extracellular matrix is thought to activate the oxidation-reduction system, leading to an enhanced production of free radicals resulting in increased oxidative stress and fibrosis [49].

\subsection{Stem Cell Involvement}

A recent study has proposed that diabetic cardiomyopathy may be a stem cell disease. Increased oxidative stress in diabetes can alter cardiac progenitor cell (CPC) func- 
tion, leading to defective cardiac progenitor cell growth and myocyte formation, causing premature myocardial aging, apoptosis and heart failure. It was also noted that cardiac progenitor cell apoptosis and heart failure were ameliorated by ablation of the p66shc gene in an animal model [50].

\section{CLINICAL PRESENTATION}

Diabetic cardiomyopathy presents with LVH and/or systolic \& diastolic dysfunction which can be categorised into 4 stages depending on the symptoms.

\subsection{Diastolic Dysfunction in Diabetes}

LV Diastolic dysfunction is much more common than initially reported in subjects with well-controlled type 2 diabetes who are asymptomatic for myocardial disease. In a study of well-controlled type II diabetic patients without any evidence of diabetic complications, hypertension, coronary artery disease, congestive heart failure, thyroid or overt renal disease, LV diastolic dysfunction was present in $60 \%$ of subjects, of whom $28 \%$ had a pseudonormal pattern of ventricular filling (indicating raised filling pressure), and 32\% had impaired relaxation [51]. A similar study of young type I diabetic patients without known cardiac disease demonstrated reduced early and increased late peak mitral velocity, as well as prolonged deceleration time and isovolumic relaxation time compared with controls, despite normal LV dimensions and systolic function [52]. Some studies comparing type I and type II diabetes have reported that preclinical myocardial disease is more prevalent in type II diabetes [53, 54].

\subsection{Systolic Dysfunction in Diabetes}

In the context of diabetic cardiomyopathy, systolic dysfunction occurs late, often when patients have already developed significant diastolic dysfunction. Studies have reported that many of those who have normal LV systolic function at rest may show abnormalities during exercise or dobutamine stress [55,56], indicating that LV systolic reserve is reduced in these patients. Diabetic patients have been shown to have a lower cardiac output during supine exercise than controls, with no difference at rest mainly due to a lower stroke volume [57].

It has been suggested that an abnormal EF response during exercise may be due to alterations in ventricular loading conditions and/or cardiac autonomic innervation rather than to abnormalities of contractility itself. Despite subgroups showing an abnormal EF response to exercise, all patients with diabetes had a normal response to afterload manipulation, normal baseline ventricular contractility as assessed by load- and heart rate-independent endsystolic indexes and normal contractile reserve, as asse- ssed with dobutamine challenge [58].

\section{STAGES OF DIABETIC CARDIOMYOPATHY}

Clinical symptoms of heart failure such as class 1 dyspnoea, according to the New York Heart Association (NYHA), can be absent at an early stage or may be very mild. Maisch et al. [59] have recently proposed the following classification of diabetic cardiomyopathy based on the clinical phenotypes:

\subsection{Stage 1 Diabetic Cardiomyopathy}

Heart failure with preserved EF (HFPEF) in diabetic patients often associated with hypertrophy without relevant hypertension. Relevant CAD, valvular disease and uncontrolled hypertension are not present.

It is the earliest form of diabetic cardiomyopathy and can be detected in $75 \%$ of asymptomatic diabetic patients [10].

\subsection{Stage 2 Diabetic Cardiomyopathy}

Systolic and diastolic heart failure with dilatation and reduced ejection (HFREF) in diabetic patients excluding relevant CAD, valvular disease and uncontrolled hypertension, although $\mathrm{CAD}$ and hypertension could play a minor role. Myocardial infarction or uncontrolled hypertension should not be present.

\subsection{Stage 3 Diabetic Cardiomyopathy}

Systolic and/or diastolic heart failure in diabetic patients with small vessel disease (microvascular disease) and/or microbial infection and/or inflammation and/or hypertension but without CAD. Hypertension, microangiopathy and viral heart disease with or without myocarditis can be the contributing factors.

\subsection{Stage 4 Diabetic Cardiomyopathy}

If heart failure may also be attributed to infarction or ischemia and remodelling in addition to stage 3 , the term should be heart failure in diabetes or stage 4 diabetic cardiomyopathy.

\section{MANAGEMENT OF DIABETIC CARDIOMYOPATHY}

\subsection{Glycaemic Control}

Prompt and appropriate treatment of diabetes is clinically relevant because of its role in the pathogenesis of heart failure. Although there is some data suggesting that poor glycaemic control contributes to myocardial dysfunction, evidence that improvements in glycaemic control are therapeutic are limited. The UKPDS (UK Prospective Dia- 
betes Study) failed to show a significant benefit of intensive blood glucose control using either sulphonylureas or insulin on the risk of developing macrovascular disease in patients with Type II diabetes [60]. This study however had significant methodological limitations which merit consideration when interpreting the results [61]. These include that the study was unblinded and continued when no difference was observed at the initial agreed time point for analysis, patients in the diet-only group actually received drug treatment if the fasting plasma glucose was $>15 \mathrm{mmol} / \mathrm{l}$, and at 9 years only $25 \%$ of patients were on monotherapy.

Hansen et al. [62] more recently showed that both myocardial function and myocardial blood volume were reduced in patients with insulin dependent diabetes, and after administration of $\mathrm{C}$ peptide a $12 \%$ improvement of function was seen in association with improvements of myocardial blood volume and flow. Similarly in another study, von Bibra et al. [63] reported improvements of myocardial function and perfusion with insulin. They also noted that the degree of both mechanical change and perfusion was related to the degree of change of fasting insulin with treatment.

There is some data suggesting that diabetic cardiomyopathy does not develop in patients with tightly controlled type 1 diabetes, supporting an important role for hyperglycaemia in the pathogenesis of diabetic cardiomyopathy [64].

There is not much data currently on the choice of glucose lowering agents in diabetic cardiomyopathy. Glucagon-like peptide-1 analogues have been associated with improvement in hemodynamic variables in diabetic patients without overt heart failure [65]. Improved cardiac parameters were also noted with this agent class post-infarction and in advanced heart failure. Use of thiazolidinediones in the management of patients with diabetic cardiomyopathy is difficult due to complications with fluid overload. As a consequence of unforeseen increase in cardiovascular mortality with thiazolidinedoines therapy, US FDA now mandates that all new hypoglycaemic agents should be trialled for diabetic population.

In general, the choice of glucose lowering approach should be directed towards clinical characteristics, such as the presence or absence renal dysfunction, risk of hypoglycaemia, age, volume status, and concomitant drug therapy.

\subsection{Blood Pressure Control}

No specific data related to changes of myocardial function in diabetes with improved blood pressure control are available. However improvement in mortality was observed with tight blood pressure control in the UKPDS trial [66], with a $15 \%$ reduction of mortality for every 10 mm Hg reduction of systolic blood pressure.

\subsection{Treatment of Fibrosis}

The important role of the RAAS in the pathogenesis of complications in diabetic patients has been discussed above. There is evidence suggesting that angiotensin converting enzyme inhibitors (ACE inhibitors) can prevent myocardial fibrosis, cardiac hypertrophy, and myocardial mechanical dysfunction associated with diabetic cardiomyopathy [67-69]. ACE inhibitors and angiotensin-1 receptor blocking agents have also been shown to prevent coronary perivascular fibrosis and collagen deposition [70,71]. Evidence also suggests a beneficial effect of aldosterone antagonism in diastolic heart failure by its effects on cardiac hypertrophy and fibrosis [72,73].

\subsection{Cross Link Breakers}

Fibrillar proteins, such as collagens type I and III, and elastin form an intricate widespread network in the extracellular matrix and provide a basis for maintaining the physical structure of the heart and vessels as well as cardiovascular function. Collagen and elastin fibres are enzymatically cross-linked to form matrix.

In addition to these enzymatically formed cross-links, collagen fibres may be linked non-enzymatically, most notably by formation of advanced glycation end-products (AGEs). In addition to diabetes as mentioned earlier, AGEs are also formed increasingly in hypertension and they accumulate with aging. Various effects of AGEs on cardiovascular structure and function have been described above, forming the basis of breaking AGEs (e.g. alanine aminotransferase 711) as a potential tool in the therapy of cardiovascular injury related to diabetes, hypertension and aging.

Limited animal and human data indicate benefit with cross-link breakers. In studies of aging non-diabetic dogs, cross-link breakers caused a significant reduction (approximately 40\%) in left ventricular stiffness, which was accompanied by improvement in cardiac function [74].

Another study in diabetic dogs, cross-link breakers restored LV ejection fraction, reduced aortic stiffness and $\mathrm{LV}$ mass with no reduction in blood glucose level, and reversed the up regulation of collagen type I and type III [75].

\subsection{Other Novel Therapies Targeting Diabetic Cardiomyopathy}

In addition to the above mentioned cross-link breakers, other therapies directed toward prevention and progresssion of diabetic cardiomyopathy targeting either enhanced fibrosis/collagen deposition or alterations in cardiomyocyte metabolism which are still in experimental stages include AGEs inhibitors (e.g. aminoguanidine, alani- 
ne aminotransferase 946, and pyridoxamine), copper chelation therapy (e.g. trientine), and modulators of free fatty acid metabolism (e.g. trimetazidine).

Modulators of free fatty acid metabolism have proven useful in the management of angina, but their efficacy on diabetic cardiomyopathy is unknown. Newer agents like Exenatide (recombinant glucagon-like peptide-1,) or Sitagliptin (DPP4 inhibitor) are yet to be studied specifically in patients with diabetic cardiomyopathy despite promising cardiac effects with glucagon-like peptide-1 infusion in mechanistic studies.

\section{CONCLUSION}

Diabetic cardiomyopathy is common and can present with symptomatic or asymptomatic myocardial dysfunction. With the rising prevalence of diabetes, it has major financial and health implication on the healthcare systems. Many newer treatment targets are being explored showing promising initial results, however, further trials are needed for proven benefits. Careful glycaemic control and early administration of neurohormonal antagonists currently remain the mainstay of therapy.

\section{REFERENCES}

[1] Marwick, T.H. (2008) Diabetic heart disease. Postgraduate Medical Journal, 84, 188-192. doi:10.1136/hrt.2005.067231

[2] Aneja, A., et al. (2008) Diabetic cardiomyopathy: Insights into pathogenesis, diagnostic challenges, and therapeutic options. American Journal of Medicine, 121, 748-757. doi:10.1016/j.amjmed.2008.03.046

[3] Aronow, W.S. and Ahn, C. (1999) Incidence of heart failure in 2737 older persons with and without diabetes mellitus. Chest, 115, 867-868. doi:10.1378/chest.115.3.867

[4] Bella, J.N., et al. (2001) Separate and joint effects of systemic hypertension and diabetes mellitus on left ventricular structure and function in American Indians (the Strong Heart Study). American Journal of Cardiology, 87, 1260-1265. doi:10.1016/S0002-9149(01)01516-8

[5] Bertoni, A.G., et al. (2006) Diabetic cardiomyopathy and subclinical cardiovascular disease: The Multi-Ethnic Study of Atherosclerosis (MESA). Diabetes Care, 29, 588594. doi:10.2337/diacare.29.03.06.dc05-1501

[6] Bertoni, A.G., et al. (2003) Diabetes and idiopathic cardiomyopathy: A nationwide case-control study. Diabetes Care, 26, 2791-2795. doi:10.2337/diacare.26.10.2791

[7] Nichols, G.A., et al. (2001) Congestive heart failure in type 2 diabetes: Prevalence, incidence, and risk factors. Diabetes Care, 24, 1614-1619. doi:10.2337/diacare.24.9.1614

[8] Kannel, W.B., Hjortland, M. and Castelli, W.P. (1974) Role of diabetes in congestive heart failure: The Framingham study. American Journal of Cardiology, 34, 29-34. doi:10.1016/0002-9149(74)90089-7
[9] Iribarren, C., et al. (2001) Glycemic control and heart failure among adult patients with diabetes. Circulation, 103, 2668-2673. doi:10.1161/01.CIR.103.22.2668

[10] Boyer, J.K., et al. (2004) Prevalence of ventricular diastolic dysfunction in asymptomatic, normotensive patients with diabetes mellitus. American Journal of Cardiology, 93, 870-875. doi:10.1016/j.amjcard.2003.12.026

[11] Barrett-Connor, E. and Ferrara, A. (1998) Isolated postchallenge hyperglycemia and the risk of fatal cardiovascular disease in older women and men. The Rancho Bernardo Study. Diabetes Care, 21, 1236-1239. doi:10.2337/diacare.21.8.1236

[12] Lowe, L.P., et al. (1997) Diabetes, asymptomatic hyperglycemia, and 22-year mortality in black and white men. The Chicago Heart Association Detection Project in Industry Study. Diabetes Care, 20, 163-169. doi:10.2337/diacare.20.2.163

[13] Barzilay, J.I., et al. (1994) Coronary artery disease and coronary artery bypass grafting in diabetic patients aged $>$ or $=65$ years (report from the Coronary Artery Surgery Study [CASS] Registry). American Journal of Cardiology, 74, 334-339. doi:10.1016/0002-9149(94)90399-9

[14] Waller, B.F., et al. (1980) Status of the coronary arteries at necropsy in diabetes mellitus with onset after age 30 years. Analysis of 229 diabetic patients with and without clinical evidence of coronary heart disease and comparison to 183 control subjects. American Journal of Medicine, 69, 498-506. doi:10.1016/S0149-2918(05)80002-5

[15] Granger, C.B., et al. (1993) Outcome of patients with diabetes mellitus and acute myocardial infarction treated with thrombolytic agents. The Thrombolysis and Angioplasty in Myocardial Infarction (TAMI) Study Group. Journal of the American College of Cardiology, 21, 920-925. doi:10.1016/0735-1097(93)90348-5

[16] Mueller, H.S., et al. (1992) Predictors of early morbidity and mortality after thrombolytic therapy of acute myocardial infarction. Analyses of patient subgroups in the Thrombolysis in Myocardial Infarction (TIMI) trial, phase II. Circulation, 85, 1254-1264. doi:10.1161/01.CIR.85.4.1254

[17] Stein, B., et al. (1995) Influence of diabetes mellitus on early and late outcome after percutaneous transluminal coronary angioplasty. Circulation, 91, 979-989. doi:10.1161/01.CIR.91.4.979

[18] Vigorito, C., et al. (1980) Severity of coronary artery disease in patients with diabetes mellitus. Angiographic study of 34 diabetic and 120 nondiabetic patients. American Heart Journal, 100, 782-787. doi:10.1016/0002-8703(80)90056-3

[19] Jaffe, A.S., et al. (1984) Increased congestive heart failure after myocardial infarction of modest extent in patients with diabetes mellitus. American Heart Journal, 108, 3137. doi:10.1016/0002-8703(84)90541-6

[20] Stone, P.H., et al. (1989) The effect of diabetes mellitus on prognosis and serial left ventricular function after acute myocardial infarction: Contribution of both coronary disease and diastolic left ventricular dysfunction to the adverse prognosis. The MILIS Study Group. Journal of the American College of Cardiology, 14, 49-57. 


\section{doi:10.1016/0735-1097(89)90053-3}

[21] Kouvaras, G., Cokkinos, D. and Spyropoulou, M. (1988) Increased mortality of diabetics after acute myocardial infarction attributed to diffusely impaired left ventricular performance as assessed by echocardiography. Japan Heart Journal, 29, 1-9. doi:10.1536/ihj.29.1

[22] Nitenberg, A., et al. (1993) Impairment of coronary vascular reserve and ACh-induced coronary vasodilation in diabetic patients with angiographically normal coronary arteries and normal left ventricular systolic function. Diabetes, 42, 1017-1025. doi:10.2337/diabetes.42.7.1017

[23] Nahser Jr., P.J., et al. (1995) Maximal coronary flow reserve and metabolic coronary vasodilation in patients with diabetes mellitus. Circulation, 91, 635-640. doi:10.1161/01.CIR.91.3.635

[24] Factor, S.M., et al. (1981) Hypertensive-diabetic cardiomyopathy in the rat: An experimental model of human disease. American Journal of Pathology, 102, 219-228.

[25] Factor, S.M., Minase, T. and Sonnenblick, E.H. (1980) Clinical and morphological features of human hypertensivediabetic cardiomyopathy. American Heart Journal, 99, 446-458. doi:10.1016/0002-8703(80)90379-8

[26] Kawaguchi, M., et al. (1997) A comparison of ultrastructural changes on endomyocardial biopsy specimens obtained from patients with diabetes mellitus with and without hypertension. Heart Vessels, 12, 267-274. doi:10.1007/BF02766802

[27] Singh, R., et al. (2001) Advanced glycation end-products: A review. Diabetologia, 44, 129-146. doi:10.1007/s001250051591

[28] Rosen, P., Du, X. and Tschope, D. (1998) Role of oxygen derived radicals for vascular dysfunction in the diabetic heart: Prevention by alpha-tocopherol? Molecular and Cellular Biochemistry, 188, 103-111. doi:10.1023/A:1006876607566

[29] Devereux, R.B., et al. (2000) Impact of diabetes on cardiac structure and function: The strong heart study. Circulation, 101, 2271-2276. doi:10.1161/01.CIR.101.19.2271

[30] Young, M.E., McNulty, P. and Taegtmeyer, H. (2002) Adaptation and maladaptation of the heart in diabetes: Part II: Potential mechanisms. Circulation, 105, 18611870. doi:10.1161/01.CIR.0000012467.61045.87

[31] Taegtmeyer, H. (2004) Cardiac metabolism as a target for the treatment of heart failure. Circulation, 110, 894-896. doi:10.1161/01.CIR.0000139340.88769.D5

[32] Rodrigues, B., Cam, M.C. and McNeill, J.H. (1998) Metabolic disturbances in diabetic cardiomyopathy. Molecular and Cellular Biochemistry, 180, 53-57. doi:10.1023/A:1006882805197

[33] Boudina, S. and Abel, E.D. (2006) Mitochondrial uncoupling: A key contributor to reduced cardiac efficiency in diabetes. Physiology (Bethesda), 21, 250-258. doi:10.1152/physiol.00008.2006

[34] Tarquini, R., et al. (2011) The diabetic cardiomyopathy. Acta Diabetologica, 48, 173-181. doi:10.1007/s00592-010-0180-x

[35] Tesfamariam, B., Brown, M.L. and Cohen, R.A. (1991) Elevated glucose impairs endothelium-dependent relaxa- tion by activating protein kinase C. Journal of Clinical Investigation, 87, 1643-1648. doi:10.1172/JCI115179

[36] Tesfamariam, B., Jakubowski, J.A. and Cohen, R.A. (1989) Contraction of diabetic rabbit aorta caused by endothelium-derived PGH2-TxA2. The American Journal of Physiology, 257, H1327-H1333.

[37] Fein, F.S. and Sonnenblick, E.H. (1985) Diabetic cardiomyopathy. Progress in Cardiovascular Diseases, 27, 255-270.

[38] Neumann, S., et al. (2002) Aldosterone and D-glucose stimulate the proliferation of human cardiac myofibroblasts in vitro. Hypertension, 39, 756-760. doi:10.1161/hy0302.105295

[39] Liu, Y., et al. (1995) Hypoxia regulates vascular endothelial growth factor gene expression in endothelial cells. Identification of a 5' enhancer. Circulation Research, 77, 638-643. doi:10.1161/01.RES.77.3.638

[40] Cagliero, E., et al. (1991) Characteristics and mechanisms of high-glucose-induced overexpression of basement membrane components in cultured human endothelial cells. Diabetes, 40, 102-110.

[41] Cockcroft, J.R., Webb, D.J. and Wilkinson, I.B. (2000) Arterial stiffness, hypertension and diabetes mellitus. Journal of Human Hypertension, 14, 377-380. doi:10.1038/sj.jhh.1001023

[42] London, G.M. and Guerin, A.P. (1999) Influence of arterial pulse and reflected waves on blood pressure and cardiac function. American Heart Journal, 138, 220-224. doi:10.1016/S0002-8703(99)70313-3

[43] Ohtsuka, S., et al. (1996) Alterations in left ventricular wall stress and coronary circulation in patients with isolated systolic hypertension. Journal of Hypertension, 14, 1349-1355. doi:10.1097/00004872-199611000-00014

[44] Airaksinen, K.E., et al. (1989) Augmentation of atrial contribution to left ventricular filling in IDDM subjects as assessed by Doppler echocardiography. Diabetes Care, 12, 159-161. doi:10.2337/diacare.12.2.159

[45] Monteagudo, P.T., et al. (2000) Influence of autonomic neuropathy upon left ventricular dysfunction in insulindependent diabetic patients. Clinical Cardiology, 23, 371375. doi:10.1002/clc. 4960230513

[46] Walter Jr., R.M., et al. (1991) Copper, zinc, manganese, and magnesium status and complications of diabetes mellitus. Diabetes Care, 14, 1050-1056. doi:10.2337/diacare.14.11.1050

[47] Islam, K.N., et al. (1995) Fragmentation of ceruloplasmin following non-enzymatic glycation reaction. The Journal of Biochemistry, 118, 1054-1060. doi:10.1093/jb/118.5.1054

[48] Argirova, M.D. and Ortwerth, B.J. (2003) Activation of protein-bound copper ions during early glycation: Study on two proteins. Archives of Biochemistry and Biophysics, 420, 176-184. doi:10.1016/j.abb.2003.09.005

[49] Yim, M.B., et al. (2001) Protein glycation: Creation of catalytic sites for free radical generation. Annals of the New York Academy of Sciences, 928, 48-53. doi:10.1111/j.1749-6632.2001.tb05634.x

[50] Rota, M., et al. (2006) Diabetes promotes cardiac stem 
cell aging and heart failure, which are prevented by deletion of the $\mathrm{p}^{\mathrm{shc}}$ gene. Circulation Research, 99, 42-52. doi:10.1161/01.RES.0000231289.63468.08

[51] Poirier, P., et al. (2001) Diastolic dysfunction in normotensive men with well-controlled type 2 diabetes: Importance of maneuvers in echocardiographic screening for preclinical diabetic cardiomyopathy. Diabetes Care, 24, 5-10. doi:10.2337/diacare.24.1.5

[52] Schannwell, C.M., et al. (2002) Left ventricular diastolic dysfunction as an early manifestation of diabetic cardiomyopathy. Cardiology, 98, 33-39. doi:10.1159/000064682

[53] Robillon, J.F., et al. (1994) Abnormalities suggestive of cardiomyopathy in patients with type 2 diabetes of relatively short duration. Diabete and Metabolisme, 20, 473480.

[54] Astorri, E., et al. (1997) Isolated and preclinical impairment of left ventricular filling in insulin-dependent and non-insulin-dependent diabetic patients. Clinical Cardiology, 20, 536-540. doi:10.1002/clc.4960200606

[55] Vered, A., et al. (1984) Exercise-induced left ventricular dysfunction in young men with asymptomatic diabetes mellitus (diabetic cardiomyopathy). The American Journal of Cardiology, 54, 633-637. doi:10.1016/0002-9149(84)90263-7

[56] Carlstrom, S. and Karlefors, T. (1970) Haemodynamic studies on newly diagnosed diabetics before and after adequate insulin treatment. British Heart Journal, 32, 355-358. doi:10.1136/hrt.32.3.355

[57] Karlefors, T. (1966) Haemodynamic studies in male diabetics. Acta Medica Scandinavica, 449, 45-80.

[58] Borow, K.M., et al. (1990) Myocardial mechanics in young adult patients with diabetes mellitus: Effects of altered load, inotropic state and dynamic exercise. Journal of the American College of Cardiology, 15, 1508-1517. doi:10.1016/0735-1097(90)92818-M

[59] Maisch, B., Alter, P. and Pankuweit, S. (2011) Diabetic cardiomyopathy-Fact or fiction? Herz, 36, 102-115. doi:10.1007/s00059-011-3429-4

[60] UK Prospective Diabetes Study (UKPDS) Group (1998) Intensive blood-glucose control with sulphonylureas or insulin compared with conventional treatment and risk of complications in patients with type 2 diabetes (UKPDS 33). The Lancet, 352, 837-853. doi:10.1016/S0140-6736(98)07019-6

[61] McCormack, J. and Greenhalgh, T. (2000) Seeing what you want to see in randomised controlled trials: Versions and perversions of UKPDS data. United Kingdom prospective diabetes study. British Medical Journal, 320, 1720-1723.doi:10.1136/bmj.320.7251.1720

[62] Hansen, A., et al. (2002) C-peptide exerts beneficial effects on myocardial blood flow and function in patients with type 1 diabetes. Diabetes, 51, 3077-3082. doi:10.2337/diabetes.51.10.3077

[63] H. von Bibra, et al. (2004) Augmented metabolic control improves myocardial diastolic function and perfusion in patients with non-insulin dependent diabetes. Heart, 90, 1483-1484. doi:10.1136/hrt.2003.020842
[64] Konduracka, E., et al. (2007) Diabetes-specific cardiomyopathy in type 1 diabetes mellitus: No evidence for its occurrence in the era of intensive insulin therapy. European Heart Journal, 28, 2465-2471. doi:10.1093/eurheartj/ehm361

[65] Thrainsdottir, I., et al. (2004) Initial experience with GLP-1 treatment on metabolic control and myocardial function in patients with type 2 diabetes mellitus and heart failure. Diabetes and Vascular Disease Research, 1, 40-43. doi:10.3132/dvdr.2004.005

[66] UK Prospective Diabetes Study Group (1998) Tight blood pressure control and risk of macrovascular and microvascular complications in type 2 diabetes: UKPDS 38. British Medical Journal, 317, 703-713. doi:10.1136/bmj.317.7160.703

[67] Rosen, R., Rump, A.F. and Rosen, P. (1995) The ACEinhibitor captopril improves myocardial perfusion in spontaneously diabetic (BB) rats. Diabetologia, 38, 509517. doi:10.1007/BF00400718

[68] Al-Shafei, A.I., et al. (2002) Magnetic resonance imaging analysis of cardiac cycle events in diabetic rats: The effect of angiotensin-converting enzyme inhibition. Journal of Physiology, 538, 555-572. doi:10.1113/jphysiol.2001.012857

[69] Al-Shafei, A.I., et al. (2002) Non-invasive magnetic resonance imaging assessment of myocardial changes and the effects of angiotensin-converting enzyme inhibition in diabetic rats. Journal of Physiology, 538, 541-553. doi:10.1113/jphysiol.2001.012856

[70] Zaman, A.K., et al. (2004) Salutary effects of attenuation of angiotensin II on coronary perivascular fibrosis associated with insulin resistance and obesity. Journal of $\mathrm{Mo}$ lecular and Cellular Cardiology, 37, 525-535. doi:10.1016/j.yjmcc.2004.05.006

[71] Kawasaki, D., et al. (2007) Role of activated renin-angiotensin system in myocardial fibrosis and left ventricular diastolic dysfunction in diabetic patients-Reversal by chronic angiotensin II type $1 \mathrm{~A}$ receptor blockade. Circulation Journal, 71, 524-529. doi:10.1253/circj.71.524

[72] Orea-Tejeda, A., et al. (2007) Aldosterone receptor antagonists induce favorable cardiac remodeling in diastolic heart failure patients. Revista de Investigación Clínica, 59, 103-107.

[73] Tang, W.H., et al. (2005) Aldosterone receptor antagonists in the medical management of chronic heart failure. Mayo Clinic Proceedings, 80, 1623-1630. doi:10.4065/80.12.1623

[74] Asif, M., et al. (2000) An advanced glycation endproduct cross-link breaker can reverse age-related increases in myocardial stiffness. Proceedings of National Academy of Sciences of the United States of America, 97, 2809-2813. doi:10.1073/pnas.040558497

[75] Liu, J., et al. (2003) Glycation end-product cross-link breaker reduces collagen and improves cardiac function in aging diabetic heart. American Journal of Physiology, Heart and Circulatory Physiology, 285, H2587-H2591. 